\title{
A П-ALGEBRA SPECTRAL SEQUENCE FOR FUNCTION SPACES
}

\author{
W. G. DWYER, D. M. KAN, J. H. SMITH, AND C. R. STOVER
}

(Communicated by Frederick R. Cohen)

\begin{abstract}
We construct a new kind of spectral sequence which is in some ways analogous to the unstable Adams spectral sequence. This new spectral sequence converts primary information about the homotopy groups of two spaces $K$ and $L$ into information about the homotopy groups of the function space of maps from $K$ to $L$.
\end{abstract}

\section{INTRODUCTION}

1.1. The main result. Given a map $f: K \rightarrow L$ of pointed $\mathrm{CW}$ complexes, let $\operatorname{hom}_{f}(K, L)$ denote the pointed space of pointed maps $K \rightarrow L$, with $f$ as the base point. Recall that a $\Pi$-algebra is a $(\geq 1)$-graded group with an action of the primary homotopy operations (e.g., for any pointed topological space $M$ there is a homotopy $\Pi$-algebra $\left.\pi_{*} M=\left\{\pi_{i} M\right\}_{i=1}^{\infty}\right)$. Given a map $t: X \rightarrow Y$ of $\Pi$-algebras, let $\operatorname{hom}_{t}(X, Y)$ denote the "function $\Pi$-algebra" defined in 3.4. Then (3.5) there is a natural map $b: \pi_{*} \operatorname{hom}_{f}(K, L) \rightarrow \operatorname{hom}_{\pi_{*} f}\left(\pi_{*} K, \pi_{*} L\right)$ of $\Pi$-algebras, which (3.6) is an isomorphism whenever $K$ has the homotopy type of a wedge of spheres of dimensions $\geq 1$. Our main result is a generalization of this fact. Let $\operatorname{hom}_{-}^{(p)}(-, Y) \quad(p \geq 0)$ denote the $p$ th right derived functor, in the sense of Quillen [3], of the above functor hom $-(-, Y)$ from " $\Pi$-algebras over $Y$ " to " $\Pi$-algebras".

1.2. Theorem. Let $f: K \rightarrow L$ be a map of pointed connected $C W$ complexes. Then

(1) There exists a natural second quadrant spectral sequence $\left\{E_{r}^{p, q}\right\}$ which is closely related (in the sense of $[1, \mathrm{IX}, \S 5]$ ) to $\pi_{*} \operatorname{hom}_{f}(K, L)$. The $E_{2}$-term of this spectral sequence is given by

$$
\begin{aligned}
& E_{2}^{0, q}=\operatorname{hom}_{\pi_{*} f}^{(0)}\left(\pi_{*} K, \pi_{*} L\right)_{q}=\operatorname{hom}_{\pi_{*} f}\left(\pi_{*} K, \pi_{*} L\right)_{q}, \quad q \geq 1, \\
& E_{2}^{p, q}=\operatorname{hom}_{\pi_{*} f}^{(p)}\left(\pi_{*} K, \pi_{*} L\right)_{q}, \quad q \geq p \geq 1,
\end{aligned}
$$

and the edge homomorphism

$$
\pi_{*} \operatorname{hom}_{f}(K, L) \rightarrow E_{\infty}^{0, *} \rightarrow E_{2}^{0, *}=\operatorname{hom}_{\pi_{*} f}\left(\pi_{*} K, \pi_{*} L\right)
$$

Received by the editors August 12, 1991 and, in revised form, June 1, 1992.

1991 Mathematics Subject Classification. Primary 55Q05, 55S37, 55T99.

This research was supported in part by the National Science Foundation. 
coincides with the above-mentioned map $b$.

(2) In view of 4.3 , this spectral sequence converges strongly to $\pi_{*} \operatorname{hom}_{f}(K, L)$ if $L$ has only a finite number of nontrivial homotopy groups or if the M-algebra $\pi_{*} K$ has finite cohomological dimension in the sense of 4.1

Examples of pointed connected $\mathrm{CW}$ complexes with a homotopy $\Pi$-algebra of finite cohomological dimension are ([2] and 4.4) wedges of spheres (of dimensions $\geq 1$ ) and finite-dimensional $C W$ complexes with the homotopy type of a $K(\pi, 1)$. From these one can construct other such spaces using the following lemma, which is an immediate consequence of $[2,7.5]$ and 4.4.

1.3. Lemma. If $* \rightarrow W \rightarrow X \rightarrow Y \rightarrow *$ is a short exact sequence of $\Pi$-algebras and $W$ and $Y$ have finite cohomological dimension, then so does $X$.

For example, if $K$ is a finite product of spheres or a fibration over a circle with a finite product of spheres as the fibre, then $\pi_{*} K$ has finite cohomological dimension.

1.4. Organization of the paper. We first (in $\S 2$ ) prove $1.2(1)$ only for the case in which the map $f: K \rightarrow L$ is trivial (i.e., maps all of $K$ to the base point of $L)$. In $\S 3$ we indicate what changes have to be made in order to remove this restriction.

In the last section $(\S 4)$ we observe that the abelian group $\operatorname{hom}_{t}^{(p)}(X, Y)_{q}$ can be interpreted as the $p$ th Quillen cohomology of $X$ with local coefficients in the " $q$-fold loops on $Y$ ". This immediately implies $1.2(2)$.

We would like to thank the referee for his or her comments.

\section{PRoOF of 1.2(1) (SPECIAL CASE)}

In this section we prove $1.2(1)$ for the case that the map $f: K \rightarrow L$ is trivial, i.e., $f$ maps all of $K$ to the base point $* \in L$.

We start with a brief review of the notion of a П-algebra, which involves a "category $\Pi$ of homotopy operations" which is slightly different from, although equivalent to, the one of [4].

2.1. The category $\Pi$ of homotopy operations. This will be the category which has as objects the pointed CW complexes with the homotopy type of a finite wedge of spheres of dimensions $\geq 1$ and which has as maps the homotopy classes of (pointed) maps between them. Note that

(1) the category $\Pi$ is pointed and has finite coproducts (i.e., finite wedges) but not products, and

(2) the category $\Pi$ comes with a smash functor $i: \Pi \times \Pi \rightarrow \Pi$ which sends an object $(U, V) \in \Pi \times \Pi$ to the object

$$
U \wedge V=(U \times V) /((U \times *) \vee(* \times V)) \in \Pi
$$

and which preserves coproducts in each.variable, i.e., the functors $(U \wedge-): \Pi \rightarrow$ $\Pi$ and $(-\wedge V): \Pi \rightarrow \Pi$ send coproducts to coproducts.

Using the category $\Pi$ we now define

2.2. П-algebras. Let Sets ${ }_{*}$ denote the category of pointed sets. A ח-algebra then can be defined as a contravariant functor $\Pi \rightarrow$ Sets $_{*}$ which sends coproducts to products and as a map of $\Pi$-algebras as a natural transformation between two such functors. The resulting category of $\Pi$-algebras will be denoted $\Pi$-al . 
This definition implies that, for every object $X \in \Pi$-al :

(1) $X *=*$, where * denotes the point in both categories $\Pi$ and Sets ;

(2) the values of $X$ on the objects of $\Pi$ are, up to isomorphism, determined by the values of $X$ on the spheres $S^{n} \quad(n \geq 1)$. These values will be denoted by $X_{n}$.

In view of Hilton's analysis of the homotopy groups of wedges of spheres [5, $\mathrm{XI}]$ one can thus consider a $\Pi$-algebra $X$ as a $(\geq 1)$-graded group $\left\{X_{n}\right\}_{n=1}^{\infty}$, with $X_{n}$ abelian for $n>1$, together with Whitehead product homomorphisms $[-,-]: X_{p} \otimes X_{q} \rightarrow X_{p+q-1}(p, q>1)$ and composition functors $(-) \cdot \alpha: X_{p} \rightarrow$ $X_{r}\left(\alpha \in \pi_{r} S^{p}, 1<p<r\right)$ which satisfy all the identities that hold for the Whitehead product and composition operations on the higher homotopy groups of pointed topological spaces and a left action of $X_{1}$ on $X_{n} \quad(n>1)$ which commutes with these operations. (To see that the action of $X_{1}$ on the $X_{n} \quad(n>$ 1) commutes with the other operations, suppose that $K$ is a pointed connected CW complex, for example, a wedge of circles and higher-dimensional spheres, and that $\widetilde{K}$ is the universal cover of $K$. The $\Pi$-algebra $\pi_{*} \widetilde{K}$ can be defined in a base point-free way. The action of $\pi_{1} K$ by covering transformations on $\widetilde{K}$ then induces an action of $\pi_{1} K$ on the $\Pi$-algebra $\pi_{*} \widetilde{K}$, in particular, an action commuting with all Whitehead product and composition operations. The desired result follows from the fact that this action agrees with the natural action of $\pi_{1} K$ on the higher-dimensional homotopy groups of $K$.)

An obvious example of a $\Pi$-algebra is thus provided by

2.3. The homotopy $\Pi$-algebra of a pointed topological space. Given a pointed topological space $M$, the functor $\Pi \rightarrow$ Sets $_{*}$ which sends an object $U \in \Pi$ to the set of homotopy classes of (pointed) maps $U \rightarrow M$ is easily seen to be a $\Pi$ algebra. Since (2.2), this $\Pi$-algebra is completely determined by the homotopy groups $\pi_{n} M \quad(n \geq 1)$ and the action of the (primary) homotopy operations on them, we often denote this $\Pi$-algebra by $\pi_{*} M$.

Next we define

2.4. Abelian $\Pi$-algebras. A $\Pi$-algebra $X$ will be called abelian if there exists a "multiplication map" $X \times X \rightarrow X \in \Pi$-al which turns $X$ into an abelian group object in $\Pi$-al. For every integer $n \geq 1$, the restriction $(X \times X)_{n}=$ $X_{n} \times X_{n} \rightarrow X_{n}$ then is the multiplication map for $X_{n}$ and hence (2.2) the original multiplication map on $X$, if it exists at all, is unique. A straightforward calculation now yields that a $\Pi$-algebra $X$ is abelian iff

(1) $X_{1}$ is abelian and acts trivially on the $X_{n}(n \geq 1)$,

(2) all Whitehead products in $X$ are trivial, and

(3) all composition functions in $X$ are homomorphisms.

Observe that by $[5$, p. 534] condition (3) above is redundant.

An abelian $\Pi$-algebra will be called strongly abelian if all composition functions are trivial, i.e., if it is just a $(\geq 1)$-graded abelian group.

2.5. Example. Given objects $Y \in \Pi$-al and $U \in \Pi$, let $Y^{U}: \Pi \rightarrow$ Sets $_{*}$ be the functor given by $Y^{U} V=Y(V \wedge U)$ for all $V \in \Pi$. Then it is not difficult to see that $Y^{U}$ is an abelian $\Pi$-algebra.

Using these abelian II-algebras $Y^{U}$ we can now construct 
2.6. Function $\Pi$-algebras. For two objects $X, Y \in \Pi$-al, let $\operatorname{hom}_{*}(X, Y)$ : $\Pi \rightarrow$ Sets $_{*}[4$, A.3] be the functor which sends an object $U \in \Pi$ to the set of maps $X \rightarrow Y^{U} \in \Pi$-al. Then again it is not difficult to verify that $\operatorname{hom}_{*}(X, Y)$ is an abelian $\Pi$-algebra.

If the variables in $\operatorname{hom}_{*}(-,-)$ are homotopy $\Pi$-algebras of pointed $\mathrm{CW}$ complexes (2.3) then these function $\Pi$-algebras are closely related to

2.7. Homotopy $\Pi$-algebras of function spaces. Suppose that $K$ and $L$ are pointed $\mathrm{CW}$ complexes. If $\operatorname{hom}_{*}(K, L)$ denotes the pointed space of pointed maps $K \rightarrow L$ (with the trivial map as the base point), one can construct a natural map

$$
h: \pi_{*} \operatorname{hom}_{*}(K, L) \rightarrow \operatorname{hom}_{*}\left(\pi_{*} K, \pi_{*} L\right) \in \Pi \text {-al }
$$

by sending (the homotopy class of) a map $U \rightarrow \operatorname{hom}_{*}(K, L)$ to (the homotopy class of the corresponding map $K \rightarrow \operatorname{hom}_{*}(U, L)$ and then composing the resulting map $\pi_{*} K \rightarrow \pi_{*} \operatorname{hom}_{*}(U, L) \in \Pi$-al with the isomorphism $\pi_{*} \operatorname{hom}_{*}(U, L) \cong\left(\pi_{*} L\right)^{U}$ which sends (the homotopy class of) a map $V \rightarrow \operatorname{hom}_{*}(U, L)$ to (the homotopy class of) its adjoint $V \wedge U \rightarrow L$.

A straightforward calculation now yields

2.8. Proposition. The natural map (2.7) $h: \pi_{*} \operatorname{hom}(K, L) \rightarrow \operatorname{hom}_{*}\left(\pi_{*} K, \pi_{*} L\right)$ $\in \Pi$-al is an isomorphism whenever $K$ has the homotopy type of a (not necessarily finite) wedge of spheres of dimensions $\geq 1$.

Finally, we are ready for a

2.9. Proof of 1.2(1) for $f: K \rightarrow L$ the trivial map. Let $V_{\bullet} K$ be the simplicial resolution of $K$ by wedges of spheres of dimensions $\geq 1$ described in $[4, \S 2]$. The desired second quadrant spectral sequence then will be the [1, $\mathrm{X}, \S 6]$ homotopy spectral sequence $\left\{E_{r}^{p, q}\right\}$ of the cosimplicial pointed space $\operatorname{hom}_{*}\left(V_{\bullet} K, L\right)$. If $\Delta V_{\bullet} K$ denotes the realization of $V_{\bullet} K[4, \S 3]$, then $[4, \S 3]$ the canonical map $\Delta V_{0} K \rightarrow K$ is a homotopy equivalence and [1, p. 335] $\operatorname{Tot}\left(\operatorname{hom}_{*}\left(V_{\bullet} K, L\right)\right) \cong \operatorname{hom}_{*}\left(\Delta V_{\bullet} K, L\right)$. Consequently, the spectral sequence is closely related $[1, \mathrm{IX}, \S 5]$ to $\pi_{*} \operatorname{hom}_{*}(K, L)$.

That $E_{2}^{p, q}=\operatorname{hom}_{*}^{(p)}\left(\pi_{*} K, \pi_{*} L\right)_{q}$ for $q \geq p \geq 0$ and $q \geq 1$ follows readily from $2.8,[3 ; 1, \mathrm{X}, \S 7]$, and the fact $[4, \S 2]$ that $\pi_{*} V_{\bullet} K$ is a free (and hence cofibrant) simplicial $\Pi$-algebra and its projection $\pi_{*} V_{\bullet} K \rightarrow \pi_{*} K$ onto $\pi_{*} K$ is a weak equivalence of simplicial $\Pi$-algebras.

Finally, a direct calculation shows that $\operatorname{hom}_{*}^{(0)}\left(\pi_{*} K, \pi_{*} L\right)=\operatorname{hom}_{*}\left(\pi_{*} K, \pi_{*} L\right)$ and that the edge homomorphism in the spectral sequence coincides with the map $b$ of 2.7 .

\section{Proof of 1.2(1) (General CASE)}

We now prove $1.2(1)$ without any restriction by generalizing the arguments of $\S 2$. We start with reminding the reader of the existence of

3.1. A half smash functor in $\Pi$. The category $\Pi(2.1)$ comes with a half smash functor $\Pi \times \Pi \rightarrow \Pi$ which sends an object $(U, V) \in \Pi \times \Pi$ to the object $(U \times V) /(* \times V)$ which also can be written as $U \wedge V^{+}$, where $V^{+}$denotes the pointed $\mathrm{CW}$ complex obtained from $V$ by adding a disjoint basepoint. It clearly has the following properties: 
(1) Behavior in the first variable. For every object $V \in \Pi$, the restricted functor $\left(-\wedge V^{+}\right): \Pi \rightarrow \Pi$ sends coproducts to coproducts.

(2) Behavior in the second variable. This is more complicated. For $U, V \in$ $\Pi$, there are natural maps

$$
U=U \wedge(*)^{+} \underset{q}{\stackrel{j}{\rightleftarrows}} U \wedge V^{+} \stackrel{p}{\rightarrow} U \wedge V
$$

in $\Pi$ such that $q j=\mathrm{id}$ and $p j=*$. There is also a map $k: U \wedge V \rightarrow U \wedge V^{+} \in$ $\Pi$, which is not natural, such that $p k=$ id and such that the resulting map

$$
j \vee k: U \vee(U \wedge V) \rightarrow U \wedge V^{+} \in \Pi
$$

is an isomorphism.

Next we generalize abelian $\Pi$-algebras (2.4) to

3.2. П-algebras with an action of a $\Pi$-algebra. By an action of a $\Pi$-algebra $Y$ on an abelian $\Pi$-algebra $A$ we mean a diagram in $\Pi$-al of the form

$$
* \rightarrow A \underset{c}{\rightarrow} \underset{e}{\stackrel{d}{\rightleftarrows}} Y \rightarrow *
$$

in which

(1) the right pointing arrows form an exact sequence, and

(2) $d e=\mathrm{id}: Y \rightarrow Y$.

One readily verifies that, under these conditions, the multiplication map of $A$ (2.4) turns the map $d: B \rightarrow Y$ into an abelian group object in the over category $\Pi$-al/ $Y$ (which has as objects the maps $X \rightarrow Y \in \Pi$-al and as maps the obvious commutative triangles).

3.3. Examples. Given objects $Y \in \Pi$-al and $U \in \Pi$, there is a natural action of $Y$ on the abelian (2.4) $\Pi$-algebra $Y^{U}$ given by the diagram

$$
* \rightarrow Y^{U} \stackrel{p^{*}}{\rightarrow} Y^{U^{+}} \underset{q^{*}}{\stackrel{j^{*}}{\rightleftarrows}} Y \rightarrow *
$$

in which $p, q$, and $j$ are as in 3.1 and $Y^{U^{+}}$denotes the $\Pi$-algebra such that $Y^{U^{+}}(V)=Y\left(V \wedge U^{+}\right)$for all $V \in \Pi$.

Using this natural action we now construct

3.4. Function $\Pi$-algebras. For a map $t: X \rightarrow Y \in \Pi$-al let $\operatorname{hom}_{t}(X, Y): \Pi \rightarrow$ Sets be $_{*}$ the functor which sends an object $U \in \Pi$ to the set of the maps $X \rightarrow Y^{U^{+}} \in \Pi$-al (3.3) whose composition with $j^{*}$ is $t$, pointed by the map $q^{*} t$. Then it is not difficult to verify that $\operatorname{hom}_{t}(X, Y)$ is an abelian $\Pi$-algebra. If $t$ is the trivial map, this construction clearly reduces to the one of 2.6.

As before (2.7) these function $\Pi$-algebras are related to

3.5. Homotopy $\Pi$-algebras of function spaces. If $f: K \rightarrow L$ is a map of pointed $\mathrm{CW}$ complexes and $\operatorname{hom}_{f}(K, L)$ denotes the pointed space of pointed maps $K \rightarrow L$, with the map $f: K \rightarrow L$ as base point, one can construct a natural map

$$
b: \pi_{*} \operatorname{hom}_{f}(K, L) \rightarrow \operatorname{hom}_{\pi_{*} f}\left(\pi_{*} K, \pi_{*} L\right) \in \Pi \text {-al }
$$


by sending (the homotopy class of) a map $U \rightarrow \operatorname{hom}_{f}(K, L)$ to (the homotopy class of) the corresponding map $K \rightarrow \operatorname{hom}_{*}\left(U^{+}, L\right)$ and then composing the resulting map $\pi_{*} K \rightarrow \pi_{*} \operatorname{hom}_{*}\left(U^{+}, L\right) \in \Pi$-al with the isomorphism $\pi_{*} \operatorname{hom}_{*}\left(U^{+}, L\right) \cong\left(\pi_{*} L\right)^{U^{+}}$which sends (the homotopy class of) a map $V \rightarrow \operatorname{hom}_{*}\left(U^{+}, L\right)$ to (the homotopy class of) its adjoint $V \wedge U^{+} \rightarrow L$.

Again a straightforward calculation yields

3.6. Proposition. The natural map (3.5) $b: \pi_{*} \operatorname{hom}_{f}(K, L) \rightarrow \operatorname{hom}_{\pi_{*} f}(K, L)$ $\in \Pi$-al is an isomorphism whenever $K$ has the homotopy type of a (not necessarily finite) wedge of spheres of dimensions $\geq 1$.

And we conclude this section with a

3.7. Proof of 1.2(1) (general case). If, in the notation of 2.9, $f^{\prime}: V_{\bullet} K \rightarrow L$ denotes the composition of $f$ with the projection $V_{\bullet} K \rightarrow K$, then the desired spectral sequence is the homotopy spectral sequence of the cosimplicial pointed space $\operatorname{hom}_{f^{\prime}}\left(V_{\bullet} K, L\right)$ (this is the same cosimplicial space as in 2.9 but with a different base point). The rest of the proof now proceeds just as in 2.9 , except that one has to use 3.6 instead of 2.8 .

\section{QUillen COHOMOLOGY OF П-ALGEBRAS}

In this last section we observe that the function $\Pi$-algebras of 3.4 are closely related to the

4.1. Quillen cohomology of $\Pi$-algebras. Given a map $g: W \rightarrow X \in \Pi$-al and an abelian $\Pi$-algebra $A$ with $X$-action

$$
* \rightarrow A \rightarrow B \stackrel{d}{\rightleftarrows} X \rightarrow *,
$$

let $H_{Q}(g, A)$ denote the associated abelian group of "derivations from $W$ to $A$ ", i.e., of maps $h: W \rightarrow B \in \Pi$-al such that $d h=g$. For every integer $p \geq 0$, the $p$ th Quillen cohomology group $H_{Q}^{p}(X ; A)$ of $X$ with local coefficients in $A$ then is the abelian group obtained by applying to the identity map of $X$ the $p$ th right derived functor (in the sense of Quillen [3]) of the functor $H_{Q}(-; A): \Pi-a l / X \rightarrow$ Abelian groups. Here as in 3.2 the category $\Pi-a l / X$ is the category of $\Pi$-algebras over $X$. We will say that $X$ has finite cohomological cimension if there is an integer $k \geq 0$ such that $H_{Q}^{q}(X ; A)=0$ for all $q \geq k$ and every abelian $\Pi$-algebra $A$ with an $X$-action.

As usual this definition of cohomology implies

4.2. Proposition. Let $X \in \Pi$-al, and let $* \rightarrow A^{\prime} \rightarrow A \rightarrow A^{\prime \prime} \rightarrow *$ be a short exact sequence of abelian $\Pi$-algebras with an $X$-action. Then there is a natural long exact sequence

$$
\begin{aligned}
0 & \rightarrow H_{Q}^{0}\left(X ; A^{\prime}\right) \rightarrow \cdots \rightarrow H_{Q}^{p}\left(X ; A^{\prime}\right) \\
& \rightarrow H_{Q}^{p}(X ; A) \rightarrow H_{Q}^{p}\left(X ; A^{\prime \prime}\right) \rightarrow H_{Q}^{p+1}\left(X ; A^{\prime}\right) \rightarrow \cdots .
\end{aligned}
$$

Another easy consequence of 4.1 is the following proposition, which immediately implies 1.2(2). 
4.3. Proposition. Let $t: X \rightarrow Y \in \Pi$-al. Then there is, for every $p \geq 0$ and $q \geq 1$, a natural isomorphism $\operatorname{hom}_{t}^{(p)}(X, Y)_{q} \cong H_{Q}^{p}\left(X, Y^{S^{q}}\right)$, where the $X$ action on $Y^{S^{q}}$ is the one induced by $t$ from the natural $Y$-action on $Y^{S^{q}}$ (3.3).

We end with remarking that the notion of finite cohomological dimension of a $\Pi$-algebra can be reduced to the same such notion for a simplicial ring. This follows readily from the next proposition and the fact that $[2, \S 8]$, at least in positive dimensions, the Quillen cohomology of a $\Pi$-algebra $X$ with local coefficients in a strongly (2.4) abelian $\Pi$-algebra is, apart from a shift in dimension, just ordinary cohomology of the simplicial ring $E F_{\bullet} X$ obtained by applying the enveloping ring functor $E[2, \S 3]$ to the standard free simplicial resolution $[2, \S 2] F_{0} X$ of $X$.

4.4. Proposition. Let $X \in \Pi$-al and $k \geq 0$ be such that $H_{Q}^{q}\left(X ; A^{\prime}\right)=0$ for all $q \geq k$ and every strongly abelian $\Pi$-algebra $A^{\prime}$ with an $X$-action. Then $H_{Q}^{q}(X ; A)=0$ for all $q \geq k+1$ and every abelian $\Pi$-algebra $A$ with an $X$-action.

Proof. Let $A$ be an abelian $\Pi$-algebra with an $X$-action and, for each $s \geq 0$, let $A^{(s)} \subset A$ denote the sub-П-algebra with an $X$-action such that $A_{n}^{(s)}=0$ for $n<s$ and $A_{n}^{(s)}=A_{n}$ for $n \geq s$. As each quotient $A^{(s)} / A^{(s+1)} \quad(s \geq 0)$ is a strongly abelian $\Pi$-algebra with an $X$-action, 4.2 implies inductively that $H_{Q}^{q}\left(X ; A^{(s)}\right)=0$ for all $s \geq 0$ and $q \geq k$. Furthermore, $A=\lim A^{(s)}$ and, hence, $A$ fits into a short exact sequence of abelian $\Pi$-algebras with an $X$-action

$$
* \rightarrow A \rightarrow \prod_{s} A^{(s)} \rightarrow \prod_{s} A^{(s)} \rightarrow * .
$$

The desired result now follows by applying 4.2 once again and noting that Quillen cohomology commutes with products.

\section{REFERENCES}

1. A. K. Bousfield and D. M. Kan, Homotopy limits, completions and localizations, Lecture Notes in Math., vol. 304, Springer, Berlin, 1972.

2. W. G. Dwyer and D. M. Kan, Homology and cohomology of П-algebras, Trans. Amer. Math. Soc. (to appear).

3. D. G. Quillen, On the (co)-homology of commutative rings, Categorical Algebra, Proc. Sympos. Pure Math., vol. 17, Amer. Math. Soc., Providence, RI, 1970, pp. 65-87.

4. C. R. Stover, A van Kampen spectral sequence for higher homotopy groups, Topology 29 (1990), 9-26.

5. G. W. Whitehead, Elements of homotopy theory, Graduate Texts in Math., vol. 61, Springer, Berlin, 1978.

Department of Mathematics, University of Notre Dame, Notre Dame, Indiana 46556 E-mail address: William.G.Dwyer.10nd.edu

Department of Mathematics, Massachusetts Institute of Technology, Cambridge, MASSACHUSETTS 02139

Department of Mathematics, Purdue University, West Lafayette, Indiana 47907

E-mail address: jhs@math.purdue.edu

Department of Mathematics, University of Chicago, Chicago, Illinois 60637

Current address: Department of Mathematics, Swarthmore College, Swarthmore, Pennsylvania 19081 